\title{
Interhemispheric spread and graded response in spreading cortical depression'
}

\author{
Dianne A. Cofoid \\ UNIVERSITY OF MISSOURI
}

\begin{abstract}
Abstraet
Bilateral electroencephalograms were taken from rats during unilateral spreading cortical depression. Different levels of brain wave activity as well as an interhemispheric spread of depression were seen as a function of the strength of the eliciting stimulus. Problem
\end{abstract}

Spreading cortical depression (SD) is characterized as a profound but reversible alteration of the spontaneous electrical activity of the cerebral cortex. However, the specific nature of $\mathrm{SD}$ remains elusive. Several learning investigators (Bures \& Buresova, 1963; Tapp, 1962; Travis \& Sparks, 1963) claim that unilateral potassium chloride (KC1) application to the exposed, intact brain elicits only unilateral depression. On the other hand, Gollander \& Ochs (1963) report that SD spreads interhemispherically following unilateral stimulation.

Furthermore, there is some question as to the onset and course of SD in relation to the eliciting stimulus effect on the electroencephalogram (EEG). Bures et al (1962) describe SD as an "all-or-none" reaction regardless of stimulus value. However, Leao (1944) and Marshall (1959) report SD to be a graded response ranging from complete disappearance of the EEG to slight alterations in the pattern. Recent behavioral research (Tapp, 1962) also suggests that there is an analog relationship between $\mathrm{KC1}$ concentration and behavioral deficit. The behavioral deficit and duration of the SD effect increased as a function of the $\mathrm{KC1}$ concentration used.

It would appear that a graded quanitity of $\mathrm{KC} 1$ may be responsible for a graded EEG and/or behavioral response. The purpose of this study was to determine the relationship between two concentrations of $\mathrm{KC1}$ (5\% and 20\%) applied unilaterally and the bilateral EEG as quantified by means of electronic analysis. Method

The Ss were 15 black hooded rats; 6 within each of the two $\mathrm{KC} 1$ groups, 2 operative controls, and 1 anesthetic control. All but the anesthetic control had identical operative and pre-experimental treatment. Bilateral trephine holes were cut posterior to bregma, leaving the dura intact. A barrier of dental cement was constructed around each trephine hole to prevent possible intermixing of fluids. Stainless steel screws (3/16 in) served as electrodes and were placed bilaterally over the occipital and frontal cortical surfaces. For the anesthetic control, scalp electrodes of silver wire were taped to the scalp. All experimental animals received a $\mathrm{KC} 1$ concentration on one hemisphere and Ringer's on the other. The operative controls received a bilateral Ringer's treatment. Bipolar ipsilateral graphic and magnetic tape recordings were taken of the EEG for $1 \mathrm{hr}$. while $\mathrm{S}$ remained under anesthetic (Relaxans - $300 \mathrm{mg} / \mathrm{cc}$ chloral hydrate and $250 \mathrm{mg} / \mathrm{cc}$ magnesium sulphate).

\section{Results and Diseussion}

By visual inspection of the graphic records, it was seen that the typical pretreatment record for the experimental animals consisted of high amplitude-low frequency bursts interspersed with low amplitude waves. The anesthetic control did not show these high-low amplitude periods. Marshall (1959) has reported that mechanically induced depression is easily elicited by a slight tap on the dura. It is probable that during the operative technique used, a mild mechanically induced depression was evoked and manifested by the high-low amplitude pattern in all the pretreatment experimental samples.

The application of $\mathrm{KC} 1$ clearly altered the pretreatment pattern. For the $20 \% \mathrm{KC1}$ group, the high-low amplitude pattern disappeared, accompanied by an immediate bilateral frequency increase and general regularity of pattern. One min. later, bilateral depression (flattening) of the EEG was obtained. With recovery (10 and $20 \mathrm{~min}$. after treatment) the spike-like waves reappeared and the low amplitude periods decreased in duration and number.

For the 5\% KC1 group, the application of $\mathrm{KC} 1$ seemed to have the effect of lengthening the duration of both the spike-like bursts and the low amplitude periods. None of these Ss showed an increase in frequency nor a general regularity in pattern consequent to $\mathrm{KC} 1$ application. With recovery, the low amplitude periods shortened in duration and the spike-like activity prevailed. Again the SD effect was bilateral in spite of the ipsilateral $\mathrm{KC} 1$ application.

The operative control Ss initially exhibited the same spike-like waves interspersed with low amplitude periods. During the course of the session, the low amplitude periods decreased in number and duration. This observation further supports the possibility of a mechanical depression effect which was comparable across all subjects.

Electronic analysis (amplitude probability density) of these same epochs revealed more subtle changes than were seen visually. Figure 1 shows that in the $20 \% \mathrm{KC} 1$ group both hemispheres depressed but the decrease in 


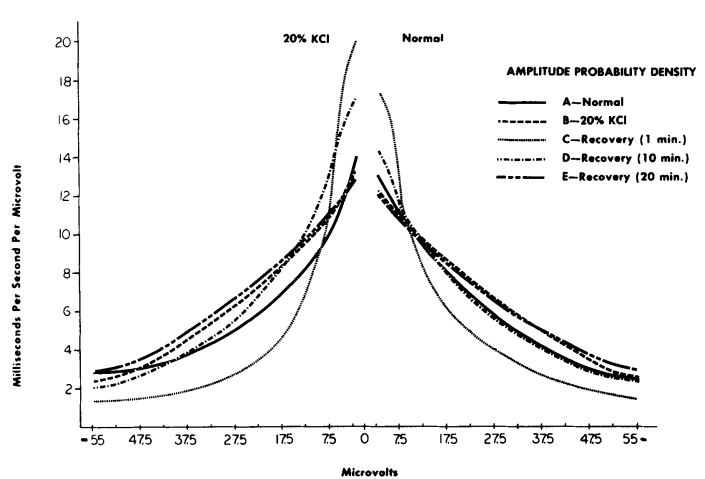

Fig. 1. Amplitude distribution for $20 \% \mathrm{KC1}$ group. Treated hemisphere (left) and non-treated hemisphere (right).

amplitude was less on the Ringer's hemisphere. With recovery, the non-treated hemisphere returned to pretreatment levels faster than the treated hemisphere. The last sample was above pretreatment levels, thus reflecting what could be seen visually as a decrease in the number of low amplitude periods among the spike-like bursts.

Different depression effects were seen in the analysis of the $5 \% \mathrm{KCl}$ group. Depression was obtained on the $\mathrm{KC1}$ hemisphere but not clearly so on the Ringer's side, and the decrease in amplitude was not as great as seen with the $20 \% \mathrm{KC} 1$ animals. However, with recovery, the EEG amplitude did return to voltages above pretreatment levels. The control data showed only increasing gains in voltages across time.

Gross visual inspection of the perfused brains revealed that no cortical damage occurred in any animals. In the two experimental groups, the dura under the $\mathrm{KC} 1$ solution showed signs of vasodilatation. Similar $\mathrm{KC1}$ effects on vascular events have been reported (Darrow \& Graf, 1945; Leao, 1944; Van Harreveld \& Ochs, 1957). It may be that the absence of vasodilatation on the untreated hemisphere indicates that the interhemispheric spread of depression cannotbe interpreted as resulting from superficial leakage of the $\mathrm{KC1}$.

The differences between these data and those reported by others could be explained by the difference in technique, the amount of cortical exposure, the effects of decreased sedation, and/or the possible wearing off of a mechanically-induced depression. However, if a mechanical depression was present at the time the chemical stimulus was introduced, the effect should have facilitated depression (Whieldon \& Van Harreveld, 1950). Since this possible effect was manifested in all animals and there was clear evidence of a difference between groups, this variable (mechanical depression) cannot be held responsible for the results or interpretation.

It may be concluded that by visual inspection and electronic analysis of the data that graded effects on cortical activity did occur as a function of $\mathrm{KC1}$ concentration. The higher $\mathrm{KC1}$ concentration (20\%) showed the greater amplitude changes and also the longest recovery time. The 5\% $\mathrm{KC1}$ group demonstrated less dramatic amplitude alterations and shorter recovery time. The decrease EEG amplitude and vasodilatation of the treated hemisphere appeared to be the common denominator of SD effects between groups. A spread of depression interhemispherically was seen clearly in the $20 \% \mathrm{KC1}$ group but less so in the $5 \%$ group.

\section{Referenees}

BURES, J., \& BURESOVA, O. Cortical spreading depression as a memory disturbing factor. J. comp. physiol. Psychol., 1963, 56, 268-272.

BURES, J., PETRAN, M., \& ZACHAR, J. Electrophysiological methods in bioelectric research. New York: Academic Press, 1962.

DARROW, C。, \& GRAF, C. Relation of electroencephalogram to photometrically observed vasomotor changes in the brain. J. Neurophysiol., 1945, 8, 449-461.

GOLLANDER, M., \& OCHS, S. Evaluation of EEG depression as an index of spreading depression in chronic preparations. Amer. Psychologist, 1963, 18, 431. (Abstract)

LEAO, A. A. P. Spreading depression of activity in the cerebral cortex. J. Neurophysiol., 1944, 7, 359-390.

MARSHALL, W. H. Spreading depression of Leao. Physiol. Rev., 1959, 39, 239-279.

TAPP, J. Reversible cortical depression and avoidance behavior in the rat. J. comp. physiol. Psychol., 1962, 55, 306-309.

TRAVIS, R., JR., \& SPARKS, D. The influence of unilateral and bilateral spreading depression during learning and subsequent relearning. J. comp. physiol. Psychol., 1963, 56, 56-59.

VAN HARREVELD, A., \& OCHS, S. Electrical and vascular concommitants of spreading depression. Amer. J. Physiol., 1957, 189, 159-166.

WIELDON, J. \& VAN HARREVELD, A. Cumulative effects of mineral cortical stimulations. EEG clin. Neurophysiol., 1950, 1, 49-57. Note

1. This research was supported by USPHS grant MH-02553, Dr. R. S. Daniel, principal investigator, and completed in partial fulfillment of the requirements for the degree of Master of Arts. I thank Professor R. S. Daniel for his valuable advice and steadfast encouragement. 